\title{
Assessment of Efficacy and Necessity of Routine Defibrillation Threshold Testing in Patients Undergoing Implantable Cardioverter-Defibrillator (ICD) Implantation
}

\author{
Sonoko Ashino, ${ }^{1}$ MD, Toshiko NAKaI, ${ }^{1}$ MD, Kazumasa Sonoda, ${ }^{1}$ MD, Naoko SASAKI, ${ }^{1}$ MD, \\ Sayaka Kurokawa, ${ }^{1}$ MD, Yukitoshi Ikeya, ${ }^{1}$ MD, Yasuo OKumura, ${ }^{1}$ MD, Kimie Ohкubo, ${ }^{1}$ MD, \\ Satoshi Kunimoto, ${ }^{1} \mathrm{MD}$, Ichiro Watanabe, ${ }^{1} \mathrm{MD}$, and Atsushi Hirayama, ${ }^{1} \mathrm{MD}$
}

\section{SUMMARY}

Defibrillation threshold (DFT) testing is performed routinely in patients undergoing implantable cardioverter-defibrillator (ICD) implantation to verify the ability of the ICD to terminate ventricular fibrillation (VF). However, neither the efficacy nor the safety of DFT testing has been proven; thus, the necessity of such testing is controversial. We conducted a retrospective study of the efficacy of DFT testing, particularly with respect to long-term outcomes of ICD implantation.

The study included 150 patients (125 men, 25 women, aged $59.0 \pm 17.6$ years) who underwent ICD or cardiac resynchronization therapy defibrillator implantation, with $(n=73)$ or without $(n=77)$ intraoperative DFT testing, between June 1996 and September 2007. VF was induced by delivery of a T-wave shock, and a 20-25-J shock was then delivered. If the 20-25-J shock failed to terminate VF, $30 \mathrm{~J}$ was delivered. We assessed whether undersensed VF events occurred during DFT testing and/or during patient follow-up and checked for any association between undersensing and delayed shock delivery. During DFT testing, fine VF was sensed, and shocks were delivered in a timely manner. Nevertheless, 2 patients in the DFT testing group died from VF within 3 years after device implantation.

DFT testing, in comparison to non-DFT testing, appeared to have no influence on the long-term outcomes of our patients, suggesting that DFT testing at the time of ICD implantation is limited. (Int Heart J 2015; 56: 618-621)

Key words: Ventricular arrhythmia, Mortality

I mplantable cardioverter-defibrillators (ICDs) have been shown to reduce mortality resulting from both ischemic $^{1,2)}$ and non-ischemic cardiomyopathies ${ }^{2)}$ and to terminate potentially fatal ventricular arrhythmias. ${ }^{3,4)}$ Current indications for an ICD include not only a near-fatal arrhythmic event $^{5)}$ and ischemic cardiomyopathy but also severe nonischemic cardiomyopathy with a low left ventricular ejection fraction (LVEF).

Defibrillation threshold (DFT) testing during ICD implantation has been considered necessary for appropriate shock delivery, ${ }^{6}$ and a DFT safety margin of $10 \mathrm{~J}$ is the standard recommendation. ${ }^{6,7)}$ However, neither the efficacy nor benefit of DFT testing has been proven. ${ }^{8-11)}$ Induction of ventricular fibrillation (VF) during DFT can cause hemodynamic compromise, clinical deterioration, and life-threatening cerebrovascular accidents. ${ }^{12-15)}$ Thus, we conducted a retrospective study to assess the effect of DFT testing on long-term outcomes in patients with an ICD or cardiac resynchronization therapy defibrillator (CRT-D).

\section{Methods}

Study patients: The study included 150 patients ( 125 men, 25 women) who received an ICD $(n=85)$ or CRT-D $(n=65)$ at our hospital between June 1996 and September 2007. Hospital records were obtained, from which we extracted the patient clinical characteristics, follow-up times, ICD intracardiac recordings, and clinical outcomes. The ICDs and CRT-Ds were implanted in a standard transvenous manner. We divided the patients into 2 groups, those in whom DFT testing was performed $(n=73)$ and those in whom DFT testing was not performed $(n=77)$, and compared the clinical data and outcomes. DFT testing and ICD/CRT-D programming: VF was induced by delivery of a 0.8 or $1 \mathrm{~J}$ T-wave shock. Upon detection of $\mathrm{VF}$, a defibrillation shock of 11-25 J was delivered. If this shock failed to terminate VF, 20-35 J was delivered. We checked the intracardiac recordings to identify any undersensed events during DFT testing and during follow-up, and we checked for an association between undersensing of VF events and any delay in ICD discharge.

The tachycardia detection zone was programmed accord-

From the ${ }^{1}$ Division of Cardiology, Department of Medicine, Nihon University School of Medicine, Tokyo, Japan.

Address for correspondence: Toshiko Nakai, MD, Division of Cardiology, Department of Medicine, Nihon University School of Medicine, 30-1 Oyaguchi Kamicho, Itabashi-ku, Tokyo 173-8610, Japan. E-mail: nakai,toshiko@nihon-u.ac.jp

Received for publication February 25, 2015. Revised and accepted May 21, 2015.

Released in advance online on J-STAGE November 6, 2015.

All rights reserved by the International Heart Journal Association. 
Table I. Patient Clinical Characteristics According to Study Group

\begin{tabular}{lccc}
\hline Characteristic & DFT $(n=73)$ & Non-DFT $(n=77)$ & $P$ \\
\hline Age (years), mean \pm SD & $55.4 \pm 17.9$ & $62.5 \pm 16.7$ & $<0.01$ \\
Male sex, $n(\%)$ & $62(84.9 \%)$ & $68(88.3 \%)$ & 0.42 \\
Follow-up period, months & $146 \pm 32$ & $79 \pm 15$ & $<0.001$ \\
Device, ICD/CRT-D & $67 / 6$ & $35 / 42$ & $<0.01$ \\
Prevention, $n(\%)$ & & & 0.24 \\
$\quad$ Primary prevention & $21(28.7 \%)$ & $31(40.2 \%)$ & $<0.0001$ \\
$\quad$ Secondary prevention & $52(71.2 \%)$ & $46(59.7 \%)$ & $<0.0001$ \\
Ejection fraction, $\%$ & $53.5 \pm 18.1$ & $38.8 \pm 17.0$ & $<0.0001$ \\
LVDd, mm & $53.8 \pm 10.9$ & $61.2 \pm 11.3$ & $<0.0001$ \\
LVDs, mm & $38.3 \pm 12.8$ & $49.9 \pm 14.0$ & 0.78 \\
QRS, mm & $109.3 \pm 24.5$ & $129.5 \pm 36.6$ & $10.5 \pm 5.2$ \\
RV amplitude at implant, mv & $11.2 \pm 4.3$ & & \\
\hline
\end{tabular}

Data are presented as mean \pm SD or $n(\%)$. DFT indicates patients who underwent defibrillation threshold testing; NonDFT, patients who did not undergo DFT testing; LVDd, left ventricular diastolic diameter; and LVDs, left ventricular systolic diameter.

Table II. Underlying Cardiac Disorders Per Study Group

\begin{tabular}{lccc}
\hline Type of heart disease & DFT $(n=73)$ & Non-DFT $(n=77)$ & $P$ \\
\hline Coronary artery disease & 21 & 27 & 48 \\
Idiopatic ventricular arrhythmia & 12 & 13 & 25 \\
Dilated cardiomyopathy & 5 (high-DFT1) & 13 & 18 \\
Brugada syndrome & 16 & 2 & 18 \\
Valvular disease & 2 & 4 & 6 \\
Hypertrophic cardiomyopathy & 6 & 5 & 11 \\
Other & 11 & 13 & 24 \\
\hline
\end{tabular}

"High defibrillation threshold, $n=1$. DFT indicates patients who underwent defibrillation threshold testing; and Non-DFT, patients who did not undergo DFT testing.

ing to the previously documented events for the secondary prevention patients. The ICD/CRT-D was programmed to deliver antitachycardia pacing (ATP) therapy or a low energy shock for relatively slow ventricular tachycardia (VT) $(<160 \mathrm{bpm})$, such that the first shock was set to $10 \mathrm{~J}$ over the DFT for VT or $\mathrm{VF} \geq 188 \mathrm{bpm}$. The device was programmed to deliver the maximal energy shock if the first shock failed. For patients in whom DFT testing was not performed, the ICD/CRT-D was programmed to deliver a maximal energy shock for episodes of fast VT or VF ( $\geq 188 \mathrm{bpm})$.

Statistical analysis: All values are expressed as the mean \pm SD. Between-group differences in variables were analyzed by unpaired $t$-test or chi-square test, as appropriate. SAS software version 9.3 (SAS Institute, Cary, North Carolina) was used for all statistical analyses, and $P<0.05$ was considered significant.

\section{RESUlTS}

Follow-up of all patients ranged from 6.5 to 17.7 years, with a median follow-up period of 9.3 years for surviving patients. DFT testing was performed in $48.6 \%$ of the total patients. The clinical characteristics of the patients by study group are shown in Table I. Patients in the non-DFT testing group were significantly older than patients in the DFT group; the sex distribution in the 2 groups was nearly equal. LVEF, LV diameter, and QRS width differed significantly between the groups, but this was because DFT testing was not performed in most patients who received a CRT-D. Right ventricular (RV) wave amplitude at the time of device implanta- tion did not differ between the 2 groups.

The underlying cardiac conditions of the patients are listed in Table II. The most frequent condition was ischemic heart disease $(32 \%)$, followed by dilated or hypertrophic cardiomyopathy (19.3\%), idiopathic ventricular arrhythmia (16.7\%), Brugada syndrome (12\%), and valvular disease (4\%); other underlying conditions made up $16 \%$ of cases. DFT testing was commonly performed in cases of Brugada syndrome.

In the DFT group, the first shock (11-25 J) terminated the VF in all but 1 patient, and there was no delay. In the patient with a high DFT (DFT > $30 \mathrm{~J}$ ), the RV wave amplitude upon implantation was $16.2 \mathrm{mV}$. This value was good in comparison to that $(11.2 \pm 4.3 \mathrm{mV})$ in patients for whom the first shock terminated the VF. The time from induction of VF to shock delivery during DFT testing did not differ significantly between the patient with the high DFT and the patients in whom the first shock was successful $(8.41 \pm 1.82$ seconds versus $7.7 \mathrm{sec}-$ onds, respectively). Analysis of intracardiac recordings in the high DFT case revealed no undersensing during VF, and the first shock was delivered without delay ( 7.7 seconds after detection of VF). The patient had dilated cardiomyopathy and was taking $50 \mathrm{mg}$ amiodarone at the time of DFT testing.

During the median 9.3-year follow-up period, $5(6.8 \%)$ and $4(5.1 \%)$ ventricular tachyarrhythmic events and appropriate shocks were recorded in the DFT group and non-DFT group, respectively. Two fatal arrhythmic events occurred in the DFT group. In both cases, the VF was terminated by the first shock without delay during DFT testing. In 1 case, the ICD was implanted for vasospastic angina provoking cardiopulmonary arrest, and in the other case, the device was implant- 
ed for VT related to an old myocardial infarction. However, unfortunately, neither of these patients escaped the risk of sudden death despite repeat maximum shocks. Intracardiac recordings at the time of the patients' deaths showed appropriate shocks terminated all VF but it recurred immediately and repeatedly, resulting in cardiac arrest. Both were cases of ischemic heart disease, leading us to speculate that the ischemia progressed as a result of the VF and that the VF recurred due to the ischemia in a vicious cycle that resulted in death.

\section{Discussion}

Our main study finding was that ICD therapy worked appropriately in both the DFT group and non-DFT group during the fairly long follow-up period (median, 9 years). In addition, ironically, 2 DFT group patients in whom VF was terminated promptly by a first shock and maximum shock energy with a safety margin $>10 \mathrm{~J}$, eventually died of uncontrollable VF.

DFT testing during ICD/CRT-D implantation has been recommended to confirm the ability of the programmed device and appropriate sensing of induced VF. ${ }^{6}$ However, the efficacy and benefit of DFT testing have not been proven, and the necessity of DFT testing is controversial. ${ }^{8-11)}$ Previous studies have shown that impaired left ventricular function (a low LVEF) is associated with hemodynamic complications after DFT, ${ }^{12,15)}$ and we ourselves encountered a CRT-D patient with acute myocardial infarction who died from multiple organ failure 1 day after DFT testing. ${ }^{16)}$ From a survey of 21 adult ICD implant centers, Birnie, et al reported the occurrence of 3 DFTrelated deaths, 5 DFT testing-related strokes, and 27 episodes requiring prolonged resuscitation over a period of 6.75 years. ${ }^{13)}$ In 2008, Poole, et al reported an increased risk of death for patients, regardless of whether they received appropriate or inappropriate ICD shocks, ${ }^{17)}$ and since that time, a reduction in the shock delivery burden has been recommended. Blatt, et al investigated whether DFT testing during ICD implantation predicts clinical outcomes and found no difference in shock efficacy or long-term mortality between their low DFT group (< $10 \mathrm{~J})$ and high DFT group (> $10 \mathrm{~J}){ }^{8}{ }^{8}$ Arnson, et al reported no significant differences in the incidences of mortality, malignant ventricular arrhythmias, and inappropriate ICD discharges between patients who underwent DFT testing and those who did not. ${ }^{18)}$ Stavrakis, et al, on the basis of a meta-analysis of studies involving patients followed up for a median of 24 months, also concluded that DFT testing at the time of ICD implantation does not reduce total mortality or ventricular arrhythmias. Viskin, et $a l^{19)}$ noted that by 2008 they had avoided DFT testing for quite a long time, citing a difference between inducible VF and spontaneous VF as 1 of 10 reasons for their decision. In our study, $5(6.8 \%)$ and $4(5.1 \%)$ ventricular tachyarrhythmic events and appropriate shocks were recorded in the DFT group and non-DFT group, respectively, with no significant difference between them. Of our 150 cases, only 1 was a high DFT case. Shukla, et $a l^{20)}$ showed higher NYHA functional class, lower LVEF, older age, congestive heart failure, and recent amiodarone therapy to be predictors of a high DFT, and Neuzner, et $a l^{21)}$ showed male sex, older age, lower LVEF, and amiodarone therapy to be predictors of a high DFT. Our patient with a high DFT had dilated cardiomyopathy with im- paired LV systolic function $(\mathrm{LVEF}=18.3 \%)$ and was taking $50 \mathrm{mg} /$ day amiodarone. We believe the amiodarone and low LVEF accounted, at least in part, for the high DFT in this case. Even if the number is small, high DFT cases definitely exist, so it may be reasonable to consider performing DFT testing in patients with factors suggestive of a high DFT. Moreover, patients undergoing ICD implantation for secondary prevention should be considered candidates for DFT testing. However, the DFT testing does not promise a lifesaving benefit to all patients. We documented 2 fatal arrhythmic events in our DFT testing group although appropriate shocks were delivered in both this group and our non-DFT testing group. The follow-up period is significantly different between the two groups. However, all arrhythmic events occurred within 96 months (which is within a shorter period of time than the follow-up period for the non-DFT group) after ICD/CRT-D implantation. Even if the follow-up period had ended at 96 months in the DFT group, the results would not have differed.

Study limitations: Our study was limited by its retrospective design, and thus our findings should be confirmed prospectively in a study that attempts to control for patient selection and treatment biases. Few ICD therapies were delivered in the 2 groups ( 5 and 4, respectively), so power to detect a true difference between groups as well as the safety of non-DFT testing may have been lacking. However, our clinical data are based on a long-term observation period (over 9 median years) and represent real-world ICD performance; thus, we believe our findings are clinically meaningful.

Conclusions: DFT testing in our patients did not guarantee good clinical outcomes. We did not find routine DFT testing to be beneficial in terms of long-term outcomes. Our data agree with the worldwide trend over the last decade toward deferring DFT testing. Considering the negative effects of ICD shocks in patients, DFT testing should be performed only after careful consideration.

\section{REFERENCES}

1. Moss AJ, Zareba W, Hall WJ, et al. Prophylactic implantation of a defibrillator in patients with myocardial infarction and reduced ejection fraction. N Engl J Med 2002; 346: 877-83.

2. Bardy GH, Lee KL, Mark DB, et al. Amiodarone or an implantable cardioverter-defibrillator for congestive heart failure. N Engl J Med 2995; 352: 225-37.

3. Goldenberg I, Gillespie J, Moss AJ, et al. Long-term benefit of primary prevention with an implantable cardioverter-defibrillator: an extended 8-year follow-up study of the Multicenter Automatic Defibrillator Implantation Trial II. Circulation 2010; 122: 126571.

4. Betts TR, Sadarmin PP, Tomlinson DR, et al. Absolute risk reduction in total mortality with implantable cardioverter defibrillators: Analysis of primary and secondary prevention trial data to aid risk/ benefit analysis. Europace 2013; 15: 813-9.

5. The Antiarrhythmics versus Implantable Defibrillators (AVID) Investigators. A comparison of antiarrhythmic-drug therapy with implantable defibrillators in patients resuscitated from near-fatal ventricular arrhythmia. N Engl J Med 1997; 337: 1576-83.

6. Marchlinski FE, Flores B, Miller JM, Gottlieb CD, Hargrove WC 3rd. Relation of the intraoperative defibrillation threshold to successful postoperative defibrillation with an automatic implantable cardioverter defibrillator. Am J Cardiol 1988; 62: 393-8.

7. Neuzner J, Liebrich A, Jung J, et al. Safety and efficacy of implantable defibrillator therapy with programmed shock energy at 
twice the augmented step-down defibrillation threshold: results of the prospective, randomized, multicenter low-energy Endotak trial. Am J Cardiol 1999; 83: 34D-9D.

8. Blatt JA, Poole JE, Johnson GW, et al. No benefit from defibrillation threshold testing in the SCD-HeFT (Sudden Cardiac Death in Heart Failure Trial). J Am Coll Cardiol 2008; 52: 551-6.

9. Calvi V, Duqo D, Capodanno D, et al. Intraoperative defibrillation threshold testing during implantable cardioverter-defibrillator insertion: do we really need it? Am Heart J 2010; 159: 98-102.

10. Brignole M, Occhetta E, Bongiorni MG, et al; SAFE-ICD Study Investigators. Clinical evaluation of defibrillation testing in an unselected population of 2,120 consecutive patients undergoing first implantable cardioverter-defibrillator implant. J Am Coll Cardiol 2012; 60: 981-7.

11. Stavrakis S, Patel NH, Reynolds DW. Defibrillation threshold testing does not predict clinical outcomes during long-term follow-up: a meta-analysis. Pacing Clin Electrophysiol 2013; 36: 1402-8.

12. Kim SG, Fisher JD, Choue CW, et al. Influence of left ventricular function on outcome of patients treated with implantable defibrillators. Circulation 1992; 85: 1304-10.

13. Birnie D, Tung S, Simpson C, et al. Complications associated with defibrillation threshold testing: the Canadian experience. Heart Rhythm 2008; 5: 387-90.

14. Karaoguz R, Altln ET, Atbasoglu EC, et al. Defibrillation testing and early neurologic outcome. Int Heart J 2008; 49: 553-63.
15. Steinbeck G, Dorwarth U, Mattke S, et al. Hemodynamic deterioration during ICD implant: predictors of high-risk patients. Am Heart J 1994; 127: 1064-7.

16. Nakai T, Ohkubo K, Okumura Y, et al. Risk of defibrillation threshold testing in severe heart failure patients: A case of cardiac resynchronization therapy (CRT-D) with acute myocardial infarction. J Arrhythm 2012; 28: 114-6.

17. Poole JE, Johnson GW, Hellkamp AS, et al. Prognostic importance of defibrillator shocks in patients with heart failure. N Engl J Med 2008; 359: 1009-17.

18. Arnson Y, Suleiman M, Glikson M, et al. Role of defibrillation threshold testing during implantable cardioverter-defibrillator placement: data from the Israeli ICD Registry. Heart Rhythm 2014; 11: 814-21.

19. Viskin S, Rosso R. The top 10 reasons to avoid defibrillation threshold testing during ICD implantation. Heart Rhythm 2008; 5: 391-3.

20. Shukla HH, Flaker GC, Jayam V, Roberts D. High defibrillation thresholds in transvenous biphasic implantable defibrillators: clinical predictors and prognostic implications. Pacing Clin Electrophysiol 2003; 26: 44-8.

21. Neuzner J, Bahawar H, Berkowitsch A, Michel U, Schiepper M, Pitschner HF. Clinical predictors of defibrillation energy requirements. Am J Cardiol 1997; 79: 205-6. 